\title{
Observation Guided Systematic Reduction of a Detailed Human Ventricular Cell Model
}

\author{
Tobias Gerach ${ }^{1}$, Daniel Wei $\beta^{2}$, Olaf Dössel ${ }^{1}$, Axel Loewe ${ }^{1}$ \\ ${ }^{1}$ Institute of Biomedical Engineering, Karlsruhe Institute of Technology (KIT), Karlsruhe, Germany \\ ${ }^{2}$ Institute for Applied and Numerical Mathematics, Karlsruhe Institute of Technology (KIT), \\ Karlsruhe, Germany
}

\begin{abstract}
In silico studies are often used to analyze mechanisms of cardiac arrhythmias. The electrophysiological cell models that are used to simulate the membrane potential in these studies range from highly detailed physiological models to simplistic phenomenological models.

To effectively cover the middle ground between those cell models, we utilize the manifold boundary approximation method (MBAM) to systematically reduce the widely used O'Hara-Rudy ventricular cell model (ORd) and investigate the influence of parametrization of the model as well as different strategies of choosing input quantities, further called quantities of interest (QoI).

As a result of the reduction process, we present three reduced model variants of the ORd model that only contain a fraction of the original model's ionic currents resulting in a twofold speedup in computation times compared to the original model. We find that the reduced models show similar action potential duration restitution and repolarization rates. Additionally, we are able to initialize and observe stable spiral wave dynamics on a $3 D$ tissue patch for 2 out of the 3 reduced models.
\end{abstract}

\section{Introduction}

On the one hand mathematical models of the cardiovascular system are becoming more sophisticated. On the other hand however, we can observe an increasing focus on simplified or reduced models for several components, e.g. electrophysiology or force development $[1,2]$. The systematic reduction method used by Lombardo and Rappel in [1] is based on a geometric and information theoretic approach and is called MBAM [3]. They managed to reduce a high dimensional atrial cell model through a series of reduction steps and arrive at a model with only $26 \%$ of the original model variables and $14 \%$ of the original parameters while retaining physiologically interpretable parameters, which is a major advantage compared to the more commonly used phenomenological models. However, they only reduced the model with regards to the voltage dynamics after applying an S1S2 stimulation protocol. In our opinion, this leaves some open questions concerning the importance of initial parametrization of the model and the choice of which model predictions need to be preserved to obtain a reduced cell model with similar characteristics to the original one. We investigate some of these questions by applying MBAM to the O'Hara-Rudy dynamic (ORd) cell model [4], which is one of the most widely used human ventricular cell models. We use two different initial parameter sets of the ORd model and first reduce the model equations with regards to only a single action potential (AP). Furthermore, we apply an S1S2 stimulation protocol similar to the one used in [1] and compare all the reduced model characteristics to the original model in 0Dand 3D-simulations. Specifically, we investigate action potential duration (APD) restitution, repolarization rate, and the models ability to predict meta-stable re-entry phenomena in a tissue patch.

\section{Methods}

\subsection{Manifold Boundary Approximation Method (MBAM)}

MBAM is a model reduction scheme first described in [3] and finds its application in areas including system biology, power systems and biochemistry. Principally, MBAM is applicable to any dynamical system, however, some limitations apply. For further information, please refer to $[3,5]$.

Mathematical models of dynamical systems can be parametrized by a vector $\boldsymbol{\theta} \in \mathbb{R}^{\mathrm{N}}$. If the model contains $N$ parameters, then the system measurement or QoI vector $\mathbf{y}=y_{m}(t, \boldsymbol{\theta})$ sweeps out an $N$-dimensional hyper-surface known as the model manifold $\mathcal{M}$. The goal of MBAM is to find a low dimensional approximation to $\mathcal{M}$, and therefore an effective reduction of $\mathbf{y}$, by finding its boundaries. 
MBAM is best described as an iterative four-step algorithm. First, we calculate the Fisher Information Matrix (FIM)

$$
\mathbf{I}=\mathbf{J}_{\boldsymbol{\theta}}^{\mathrm{T}} \mathbf{J}_{\boldsymbol{\theta}} \quad \text { with } \quad \mathbf{J}_{\boldsymbol{\theta}}=\partial \mathbf{y} / \partial \boldsymbol{\theta}
$$

of the model at an initial guess of the parameters $\boldsymbol{\theta}_{0}$. The eigenvalues of this matrix are typically spread evenly over many magnitudes for most dynamical systems. Since the smallest eigenvalues correspond to parameter combinations with little effect on the chosen QoIs, we can use the eigenvector $\mathbf{v}_{0}$ of the smallest eigenvalue as an initial direction on $\mathcal{M}$.

In the second step, we want to find a parametrized path $\boldsymbol{\theta}(\tau)$ through parameter space. We achieve this by numerically solving a geodesic equation on $\mathcal{M}$

$$
\frac{\partial^{2} \theta^{i}}{\partial \tau^{2}}=\sum_{j, k} \sum_{l, m}\left(I^{-1}\right)^{i l} \frac{\partial y_{m}}{\partial \theta_{l}} \frac{\partial^{2} y_{m}}{\partial \theta_{j} \partial \theta_{k}} \frac{\partial \theta^{j}}{\partial \tau} \frac{\partial \theta^{k}}{\partial \tau} .
$$

As initial values for the geodesic equation, we use the values $\boldsymbol{\theta}_{0}$ and $\mathbf{v}_{0}$. We monitor the calculated path $\boldsymbol{\theta}(\tau)$ until the boundary of $\mathcal{M}$ is reached, which is identified by the smallest eigenvalue of the FIM approaching zero and becoming much smaller than the next smallest eigenvalue. The boundary represents a limiting approximation to our model. This limitation reveals itself in either single parameters or parameter combinations approaching zero or infinity.

We then analytically evaluate this limit in the model by changing the model equations. This typically either involves the complete removal or reformulation of equations, which leads to a new model with $N-1$ parameters.

In a final step, we calibrate the QoIs $\tilde{y}_{m}(t, \phi)$ of the new model with the new and reduced parameter vector $\phi$ by minimizing

$$
\min _{\phi}\left(\frac{1}{M}\right) \sum_{m=1}^{M} \frac{\left|\tilde{y}_{m}(t, \phi)-y_{m}\left(t, \boldsymbol{\theta}_{0}\right)\right|}{\left|y_{m}\left(t, \boldsymbol{\theta}_{0}\right)\right|} .
$$

This four-step process is repeated until the approximated model cannot reproduce the original model's behaviour without significant error. For the purpose of this study, we chose to stop the reduction process when the error as defined in 3 was bigger than $10 \%$.

\subsection{Cardiac electrophysiology model}

Mathematical models of cellular electrophysiology are typically based on systems of ordinary differential equations (ODEs) in the form of

$$
\begin{aligned}
& C_{\mathrm{m}} \frac{\mathrm{d} V}{\mathrm{dt}}+I_{\text {ion }}(V, \mathbf{w}, \mathbf{c})=I_{\mathrm{ext}}(t), \\
& \frac{\mathrm{d} \mathbf{w}}{\mathrm{dt}}=m_{w}(V, \mathbf{w}, \mathbf{c}), \quad \frac{\mathrm{d} \mathbf{c}}{\mathrm{dt}}=m_{c}(V, \mathbf{w}, \mathbf{c}),
\end{aligned}
$$

where $V$ denotes the transmembrane potential between the intra- and extracellular fluid, $C_{\mathrm{m}}$ is the membrane capacitance, and $I_{\text {ext }}$ is the externally applied stimulus current. The ionic currents $I_{\text {ion }}$ are defined by the gating variables $\mathbf{w}$, which take values from 0 to 1 and regulate the transmembrane currents and in turn the ionic concentrations $\mathbf{c}$.

In this study, we use the ORd model [4] to represent human ventricular electrophysiology. Please note that we adopt the modifications to the inactivation and recovery gates of $I_{\mathrm{Na}}$ as proposed in [6] to overcome some of the original models shortcomings with regards to tissue simulations.

To prepare the model for the reduction using MBAM, we have to choose appropriate parameters $\mathbf{k}$ and assume that they have been log-transformed in the model, i.e. $\boldsymbol{\theta}=\log (\mathbf{k})$. This transformation serves 2 purposes: 1) we have to make sure that all parameters are restricted to positive values, since negative values for most of the parameters, e.g. conductivities, are un-physical; 2) we are nondimensionalizing the parameters, which is important for the correspondence between the local information (FIM) and the global structure (boundary). We run the reduction scheme as described in 2.1 with two different choices of parameter sets:

$$
\begin{aligned}
\mathbf{k}_{\mathrm{A}}= & \left(G_{\mathrm{Na}, \text { fast }}, G_{\mathrm{Na}, \text { late }}, G_{\mathrm{to}}, P_{\mathrm{Ca}}, G_{\mathrm{Kr}}, G_{\mathrm{Ks}}, G_{\mathrm{K} 1}, G_{\mathrm{NaCa}},\right. \\
& \left.P_{\mathrm{NaK}}, P_{\mathrm{Nab}}, P_{\mathrm{Cab}}, G_{\mathrm{Kb}}, G_{\mathrm{pCa}}\right)^{\mathrm{T}} \\
\mathbf{k}_{\mathrm{B}}=( & G_{\mathrm{Na}, \text { fast }}, G_{\mathrm{Na}, \text { late }}, G_{\mathrm{to}}, P_{\mathrm{Ca}}, G_{\mathrm{Kr}}, G_{\mathrm{Ks}}, G_{\mathrm{K} 1}, G_{\mathrm{NaCa}}, \\
& P_{\mathrm{NaK}}, P_{\mathrm{Nab}}, P_{\mathrm{Cab}}, G_{\mathrm{Kb}}, G_{\mathrm{pCa}}, \mathrm{CMDN}, \mathrm{TRPN}, \\
& \left.\mathrm{BSR}, \mathrm{BSL}, \mathrm{CSQN}, \alpha_{\mathrm{CaMK}}, \beta_{\mathrm{CaMK}}, K_{\mathrm{m}, \mathrm{CaMK}}, \beta_{\tau}\right)^{\mathrm{T}}
\end{aligned}
$$

For the meaning and initial values of these parameters, please refer to [4].

As QoIs, we first use a single AP at a basic cycle length of $1000 \mathrm{~ms}$ for the models with the above parametrizations. We shall call these models A.1 and B.1 representing the parametrizations $\mathrm{A}$ and $\mathrm{B}$. The voltage trace of the AP was sampled to include the upstroke, the repolarization and part of the resting membrane potential. Afterwards, we performed the reduction once more with parametrization B but including a S1-S2 stimulation protocol. We stimulated the cell model five times at a cycle length of $\mathrm{S} 1=1000 \mathrm{~ms}$ before applying a second stimulus at $\mathrm{S} 2=\{300,350,400,1000\} \mathrm{ms}$. Only the four S2 stimuli were sampled and used during reduction. We shall call this model B.2.

\subsection{Numerical methods}

Model reductions and single cell simulations were run in Python. We used backward differentiation formulas to numerically integrate the initial value problems given in (2) and (4) using an adaptive time step. To minimize (3), 
we used the L-BFGS-B optimization method proposed in [7]. Tissue simulations were run using acCELLerate [8] solving the monodomain equation. To ensure numerical convergence, the cell model and monodomain time steps were set to $1 \mu \mathrm{s}$.

\section{Results}

After carrying out the iterative reduction of the ORd model with MBAM, we were able to obtain three reduced models called A.1, B.1 and B.2. In the case of models A. 1 and B. 1 only the currents $I_{\mathrm{Na}}, I_{\mathrm{CaL}}, I_{\mathrm{CaNa}}, I_{\mathrm{CaK}}, I_{\mathrm{Kr}}$ and $I_{\mathrm{K} 1}$ remain in the model, which indicates that the reduced model still shows physical behaviour, since these currents are the main contributors to the AP in myocardial cells. Further reduction of these models will result in a significant change in the AP morphology. A noticeable difference between these models is that we changed the formulation of intracellular calcium in model B.1, meaning that $\frac{\mathrm{d}\left[\mathrm{Ca}^{2+}\right]_{\mathrm{i}}}{\mathrm{dt}}$ became zero during one of the reduction steps and $\left[\mathrm{Ca}^{2+}\right]_{\mathrm{i}}$ was clamped to $0.164 \mu \mathrm{M}$. This is only possible due to the more comprehensive parametrization. In model B.2, we additionally retain the currents $I_{\mathrm{NaCa}}, I_{\mathrm{NaK}}, I_{\mathrm{Cab}}$ and $I_{\mathrm{Kb}}$. We estimated the simulation times of the original model and each of the reduced models by computing 1000 action potentials at $1000 \mathrm{~ms}$ cycle length, which resulted in roughly a two-fold speed-up. The simulations took $55.7 \mathrm{~s}$, $35.8 \mathrm{~s}, 29.8 \mathrm{~s}$, and $32.5 \mathrm{~s}$ for the original, A.1, B.1, and B.2 models, respectively.

We further determine how accurate the reduced models can reproduce the behaviour of the original model by computing APD restitution curves for each model using a S1S2 stimulation protocol with more $\mathrm{S} 2$ stimuli than in the reduction process. We acquired $\mathrm{APD}_{90}$ by calculating the time between $(\partial V / \partial t)_{\max }$ and $90 \%$ of repolarization of each S2 stimulus after pacing the model for 5 beats with $1000 \mathrm{~ms}$ basic cycle length. The results are shown in figure 1. Although all models show restitutive behaviour, the reduced models A.1 and B.1 significantly differ from the original model. Especially for cycle lengths above $500 \mathrm{~ms}$ we observe shorter APDs in these models. At cycle lengths below $500 \mathrm{~ms}$, we find more similar behaviour in all models. Reduced model B.2 shows very similar APD 90 restitution over all cycle lengths used. The shorter APD in model B.1 can further be observed for the repolarization rates at $1000 \mathrm{~ms}$ cycle length shown in figure 2 . For models A.1 and B.2, the repolarization rate is similar compared to the original model.

Finally, we used a cross-field stimulation protocol to initiate spiral waves on a 3D patch. The snapshots of the observed dynamics are shown at $250 \mathrm{~ms}$ and $400 \mathrm{~ms}$ after the premature S2 stimulus in figure 3. Models A.1 and B.2 show qualitatively similar behaviour compared to the orig-

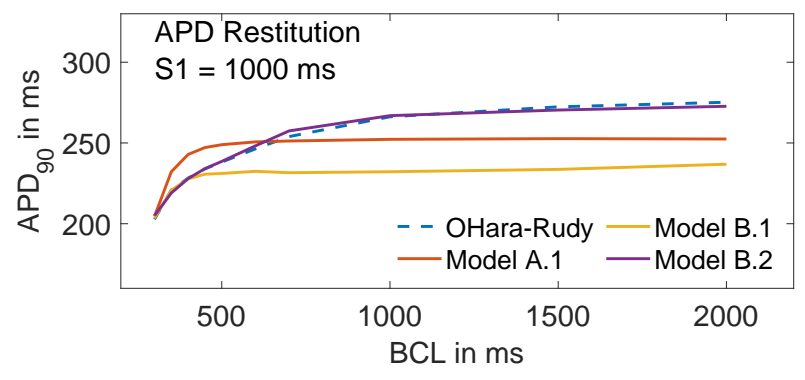

Figure 1. APD restitution of the original model and the reduced models. We acquired $\mathrm{APD}_{90}$ for $\mathrm{S} 2$ stimuli in the range from $300 \mathrm{~ms}$ to $2000 \mathrm{~ms}$ after pacing the models 5 times with $\mathrm{S} 1=1000 \mathrm{~ms}$ before each S2 stimulus.

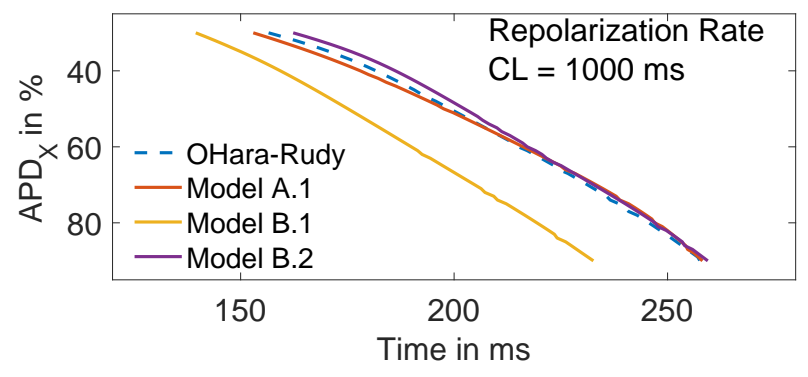

Figure 2. Repolarization rate of all four models after pacing 5 times with a cycle length of $1000 \mathrm{~ms}$.

inal ORd model. For the given simulation setup, it was not possible to initiate a spiral wave for model B.1. Additionally, we observe that the reduced models A.1 and B.1 show a slightly lower conduction velocity.

\section{Discussion}

In this study, we systematically reduced the O'HaraRudy human ventricular cell model for two different parametrizations based on the voltage trace of one action potential and a dedicated S1S2 stimulus protocol. We utilized a recently developed reduction method called MBAM, which is well suited for models consisting of a system of ODEs as present in cardiac electrophysiology models [1]. Independent of the parameter set, we were able to remove many currents from the original model including $I_{\mathrm{NaL}}, I_{\mathrm{to}}, I_{\mathrm{Ks}}, I_{\mathrm{NaCa}}, I_{\mathrm{NaK}}, I_{\mathrm{Nab}}, I_{\mathrm{Cab}}, I_{\mathrm{Kb}}, I_{\mathrm{pCa}}$, leaving only the currents which are known to be important to the morphology of the action potential. In the model B.1, the repolarization phase is mainly governed by $I_{\mathrm{CaL}}, I_{\mathrm{K} 1}$ and $I_{\mathrm{Kr}}$. As seen in figure 2, this leads to a change in repolarization rate compared to the original model. Specifically, the overall APD is up to $30 \mathrm{~ms}$ shorter and the overall repolarization is more rapid in models with parameter set $\mathrm{B}$ compared to the original model or model A.1 with parameter set $\mathrm{A}$.

Although we did not specifically take restitution dyna- 


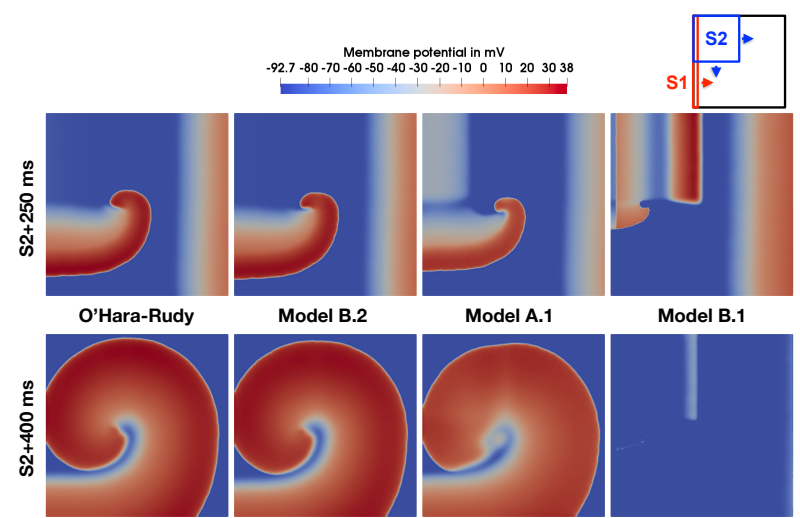

Figure 3. Snapshots of a 3D simulation using a $30 \mathrm{~mm} \times 30 \mathrm{~mm} \times 0.1 \mathrm{~mm}$ homogeneous domain discretized by tetrahedra with $0.18 \mathrm{~mm}$ average edge length. The intracellular conductivity $\sigma_{i, \mathrm{ORd}}$, A.1, B.1, B.2 $=$ $\{0.0088,0.009,0.0097,0.0088\} \mathrm{S} / \mathrm{m}$ was set to result in an isotropic propagation velocity of $133 \mathrm{~mm} / \mathrm{s}$. We used a cross-field stimulation protocol (top right picture) with two S1 stimuli at $500 \mathrm{~ms}$ followed by one S2 stimulus at $\mathrm{ERP}+150 \mathrm{~ms}$.

mics into account for all our models, they still show reasonable APD restitution behaviour. For cycle lengths of $500 \mathrm{~ms}$ and above, the reduced models A.1 and B.1 show a more flat and linear restitution curve with shorter $\mathrm{APD}_{90}$ than the original model. Model B.2, where S1S2 restitution was considered during the reduction process, is very similar to the original model.

The spiral wave dynamics of the reduced models were evaluated on a homogeneous $3 \mathrm{D}$ patch model. Using a cross-field stimulation protocol, we were able to initiate a spiral wave on all models except model B.1. This could be due to the missing formulation of the intracellular calcium concentration, which was set to a constant value during the reduction process of this model. In tissue simulations, this leads to a prolonged repolarization phase and therefore to a longer effective refractory period.

Unsurprisingly, the computation of the reduced models has become more efficient through the removal of a large portion of the equations. We note that the computation of these models is still far inferior compared to most of the purely phenomenological cell models. However, with the reduced models presented in this study, we still have a connection to physiologically relevant parameters contained in the model equations.

In conclusion, we show that fewer, more purposefully chosen parameters might be more efficient to find a reduced model with the desired behaviour. Additionally, it is sufficient to reduce the model only with regards to the transmembrane voltage to obtain a minimal model with qualitatively correct dynamic behaviour. However, inclu- ding the desired behaviour in the reduction will noticeably improve the results.

\section{Acknowledgements}

This project was funded by the Deutsche Forschungsgemeinschaft (DFG, German Research Foundation) Project-ID 258734477 - SFB 1173.

\section{References}

[1] Lombardo DM, Rappel WJ. Systematic reduction of a detailed atrial myocyte model. Chaos Woodbury NY 2017; 27(9):093914.

[2] Regazzoni F, Dedè L, Quarteroni A. Active contraction of cardiac cells: a reduced model for sarcomere dynamics with cooperative interactions. Biomechanics and modeling in mechanobiology 2018;1-24.

[3] Transtrum MK, Qiu P. Model reduction by manifold boundaries. Physical review letters 2014;113(9):098701.

[4] O'Hara T, Virag L, Varro A, Rudy Y. Simulation of the undiseased human cardiac ventricular action potential: model formulation and experimental validation. PLoS Computational Biology 2011;7(5):e1002061.

[5] Transtrum MK, Qiu P. Bridging mechanistic and phenomenological models of complex biological systems. PLoS computational biology 2016;12(5):e1004915.

[6] Passini E, Minchol A, Coppini R, Cerbai E, Rodriguez B, Severi S, Bueno-Orovio A. Mechanisms of proarrhythmic abnormalities in ventricular repolarisation and anti-arrhythmic therapies in human hypertrophic cardiomyopathy. Journal of Molecular and Cellular Cardiology 2016; 96:72 - 81. ISSN 0022-2828.

[7] Byrd RH, Lu P, Nocedal J, Zhu C. A limited memory algorithm for bound constrained optimization. SIAM Journal on Scientific Computing 1995;16(5):1190-1208.

[8] Seemann G, Sachse FB, Karl M, Weiss DL, Heuveline V, Dssel O. Framework for modular, flexible and efficient solving the cardiac bidomain equation using PETSc. Mathematics in Industry 2010;15(2):363-369.

Address for correspondence:

Tobias Gerach

Institute of Biomedical Engineering, Karlsruhe Institute of Technology (KIT), Kaiserstr. 12, 76131, Karlsruhe, Germany.

publications@ibt.kit.edu 